\title{
Same-day stereotactic aspiration and Gamma Knife surgery for cystic intracranial tumors
}

\author{
Clinical article
}

\author{
*Xiaomin Liv, M.D., Ph.D., ${ }^{1}$ Qi Yu, M.D., ${ }^{2}$ Zhiyuan Zhang, M.D., ${ }^{1}$ Yipei Zhang, M.D., ${ }^{1}$ \\ Yanhe Li, M.D., ${ }^{1}$ Dong Liu, M.D., ${ }^{1}$ Qiang Jia, M.D., Ph.D., ${ }^{3}$ Ligao Zheng, M.D., 1 \\ AND Desheng Xu, M.D. ${ }^{1}$ \\ ${ }^{I}$ Gamma Knife Center, Department of Neurosurgery, Second Hospital of Tianjin Medical University; \\ ${ }^{2}$ Graduate School of Tianjin Medical University; and ${ }^{3}$ Huanhu Hospital, Tianjin, People's Republic of China
}

\begin{abstract}
Object. The goal of this study was to evaluate the efficacy and safety of same-day stereotactic aspiration and Gamma knife surgery (GKS) for cystic intracranial tumors.

Methods. Between 1996 and 2007, 77 patients harboring cystic intracranial tumors underwent a same-day procedure of MRI-guided cyst aspiration followed by GKS. The diagnoses were metastatic tumor in 43 patients, glial tumor in 12 patients, vestibular schwannoma in 10 patients, craniopharyngioma in 9 patients, and hemangioblastoma in 3 patients.

Results. An improvement in symptoms was achieved in 68 patients $(88.3 \%)$ immediately after cyst aspiration. The mean tumor volume in this group of patients was $25.1 \mathrm{~cm}^{3}$ before aspiration and $11.1 \mathrm{~cm}^{3}$ afterward. Hemorrhage during the course of aspiration was encountered in 1 patient. Transient nausea after cyst aspiration developed in 3 patients. There was no treatment-related hematoma, seizure, neurological deficit, or infection. The median follow-up period was 16 months (range 6-108 months). Tumor control was achieved in 50 (80.6\%) of 62 patients who participated in follow-up for at least 6 months.

Conclusions. The same-day stereotactic aspiration and GKS procedure was safe in patients with cystic brain tumors. Prompt symptom relief was obtained after cyst aspiration. The decrease in tumor volume following aspiration made GKS more effective because a higher prescription dose could be administered with a lower possibility of radiation-induced side effects.
\end{abstract}

(http://thejns.org/doi/abs/10.3171/2012.7.GKS121019)

\section{KeY Words • Gamma Knife surgery • stereotactic radiosurgery cystic tumor $\bullet$ stereotaxy $\bullet$ aspiration}

$\mathrm{L}$ ARGE peritumoral and intratumoral cysts in the central nervous system are often associated with severe neurological symptoms due to their mass effect. ${ }^{1,10}$ Resection of tumor and tumor-associated cysts may provide rapid symptom relief and sometimes cure the disease. Resection is not always appropriate, however, if the lesion is located in a deep or critical area. In addition, resection is not a safe choice for patients with severe systemic diseases or advanced age, because their physical condition may be poor and not suitable for general anesthesia. ${ }^{3,16}$ For these patients, a multimodal treatment approach may be employed for the alleviation of clinical symptoms and the control of tumor growth. ${ }^{13}$

Abbreviation used in this paper: GKS = Gamma Knife surgery.

* Drs. Xiaomin Liu and Qi Yu contributed equally to this work.
Image-guided stereotactic aspiration of tumor-associated cysts is a minimally invasive procedure performed to relieve mass effect. ${ }^{4-6}$ With the aid of modern imaging techniques and improved surgical instruments, the stereotactic aspiration needle can safely reach most locations in the brain.

Gamma Knife surgery is an important therapeutic procedure used to treat intracranial lesions. ${ }^{9}$ It directs conformal radiation to the intracranial target in a single session. Large lesions are treated with a decreased prescription dose to avoid radiation-associated complications. An insufficient total dose, however, leads to an unsatisfactory outcome with respect to tumor control. Following aspiration of tumor-associated cysts, the volume of the tumor may be significantly reduced. This makes GKS safer and 
more effective. In the present study we analyzed 77 cases of tumor-associated cysts treated with same-day stereotactic aspiration and GKS to evaluate the efficacy and safeness of this combined treatment modality.

\section{Methods}

Between 1996 and 2007, 77 patients with imagingdefined cystic brain tumors were treated using same-day stereotactic cyst aspiration and GKS at the Gamma Knife Center, Department of Neurosurgery, Second Hospital of Tianjin Medical University. The patient group consisted of 41 male and 36 female patients with a median age of 49 years (range $22-75$ years). The diagnoses in this group were metastatic tumor in 43 patients, glial tumor in 12 patients, vestibular schwannoma in 10 patients, craniopharyngioma in 9 patients, and hemangioblastoma in 3 patients (Table 1). Thirty-nine patients had undergone at least one prior open resection. Eight patients had received chemotherapy at an earlier time. Presenting symptoms at the time of treatment included headache, nausea and vomiting, hemiparesis, visual deterioration, and diplopia. Informed consent was obtained from each patient.

On the morning of the treatment day, the patients' scalps were shaved. A local scalp anesthetic agent and a mild sedative was administered to each patient before a Leksell model $\mathrm{G}$ stereotactic head frame (Elekta AB) was affixed to the head. High-resolution MRI with contrast enhancement was performed after frame application. A series of T1-weighted axial images (2- to 3-mm thick slices) with no slice interval was obtained and transferred to the workstation. Leksell SurgiPlan was used for cyst volume calculation and trajectory planning.

Cyst aspiration was performed carefully after twistdrill craniotomy and catheter placement. Following stereotactic aspiration, the patients underwent an additional MRI study in which the same sequences and slice thickness were used. Tumor volume and radiation dose were calculated using Leksell Gamma Plan software (versions 3.0-5.31). Gamma Knife surgery was performed using the Leksell Gamma Knife model B and model C. The

TABLE 1: Characteristics of 77 patients who underwent stereotactic aspiration and GKS for cystic intracranial tumors

\begin{tabular}{lc}
\hline \multicolumn{1}{c}{ Characteristic } & Value \\
\hline M/F ratio & $41: 36$ \\
median age in yrs (range) & $49(22-75)$ \\
mean tumor vol in cm (range) $^{2}$ & $25.1(5.6-76.2)$ \\
previous treatment-no. of patients (\%) & \\
$\quad$ resection & $39(50.6)$ \\
chemotherapy & $8(10.4)$ \\
diagnosis-no. of patients (\%) & \\
metastatic tumor & $43(55.8)$ \\
glial tumor & $12(15.6)$ \\
vestibular schwannoma & $10(13.0)$ \\
craniopharyngioma & $9(11.7)$ \\
hemangioblastoma & $3(3.9)$ \\
\hline
\end{tabular}

median tumor margin dose was 17 Gy (range 12-20 Gy). In patients harboring more than 1 metastatic tumor, other noncystic lesions were also treated by GKS. After treatment the head frame was removed and patients were kept in the hospital for at least 3 days.

Approval for this study was obtained from the Second Hospital of Tianjin Medical University ethics board. For patients with malignant intracranial tumors MRI and clinical evaluations were scheduled at 3-month intervals after GKS; for patients with benign tumors, 6-month intervals were used for follow-up. Fifteen patients who were lost to follow-up or participated in follow-up less than 6 months were excluded from the assessment of tumor control. The evaluation of tumor control was made only for cystic tumors treated by stereotactic aspiration before GKS. The mean duration of follow-up was 16 months (range 6-108 months).

\section{Results}

The mean tumor volume in this patient group was $25.1 \mathrm{~cm}^{3}$ before aspiration and $11.1 \mathrm{~cm}^{3}$ afterward. An immediate improvement in symptoms was achieved in 68 patients $(88.3 \%)$ following cyst aspiration. A small amount of bleeding was encountered in 1 patient at the end of cyst aspiration. In that case blood was allowed to drain out of the catheter until it stopped spontaneously within 1 minute. A CT scan obtained in this patient after the aspiration procedure revealed no hematoma in the cyst. Three patients experienced transient new symptoms of nausea after aspiration, but these resolved within several hours (Table 2). There was no treatment-related hematoma, seizure, neurological deficit, or infection.

The median follow-up period was 16 months (range 6-108 months) in the 62 patients who participated in follow-up for at least 6 months. Follow-up MRI in 5 patients showed disappearance of the cystic tumor, which in these instances was a metastatic lesion. Decreases in tumor size were found in 38 patients, and stable tumor sizes were found in 7 patients during the follow-up period. Tumor progression was observed in 12 patients after GKS. Nine patients underwent later resection and 2 patients underwent repeated GKS. One patient received external beam radiotherapy (Table 3). Fourteen patients eventually required repeated aspiration and Ommaya reservoir placement.

\section{Discussion}

The underlying mechanism of tumor-associated cyst formation in the central nervous system remains unknown. Tumor degeneration, blood-brain barrier disrup-

TABLE 2: Outcomes after stereotactic cyst aspiration in 77 patients

\begin{tabular}{lc}
\hline \multicolumn{1}{c}{ Characteristic } & No. of Patients (\%) \\
\hline immediate improvement in symptoms & $68(88.3)$ \\
hemorrhage & $1(1.3)$ \\
new symptom & $3(3.9)$ \\
\hline
\end{tabular}


TABLE 3: Tumor control and further treatment in 62 patients participating in a follow-up period of at least 6 months

\begin{tabular}{lc}
\hline \multicolumn{1}{c}{ Characteristic } & Value \\
\hline median follow-up in mos (range) & $16(6-108)$ \\
tumor control—no. of patients (\%) & \\
$\quad$ total & $50(80.6)$ \\
disappearance & $5(8.1)$ \\
decreased size & $38(61.3)$ \\
stable size & $7(11.3)$ \\
tumor progression—-total no. of patients (\%) & $12(19.4)$ \\
treatment for tumor progression-no. of patients (\%) & \\
$\quad$ resection & $9(14.5)$ \\
repeated GKS & $2(3.2)$ \\
$\quad$ external beam radiotherapy & $1(1.6)$ \\
repeated aspiration \& reservoir placement & $14(22.6)$ \\
\hline
\end{tabular}

tion, intratumoral hemorrhage, and peritumoral edema are all possible causes of cyst formation..$^{2,6,15}$ On imaging studies two types of tumor-associated cysts can be identified: intratumoral cyst and peritumoral cyst. In intratumoral cysts the wall of the cyst consists of tumor cells, which obviously need treatment. ${ }^{12,18}$ In peritumoral cysts the tumor is a nodule in the cyst wall. No tumor cells reside in the cyst wall; therefore radiation targeting of the cyst wall may not confer benefit..$^{11,14,16}$ In the present study there were 4 cystic tumors with mural nodules (1 pilocytic astrocytoma and 3 hemangioblastomas). If the total volume of the tumor nodule and collapsed cyst remnant remains below the safe threshold (mean diameter < $35 \mathrm{~mm}$ ), an effective prescription dose can be administered to the lesion safely while sparing critical tissue. In this situation no harm will result from treating the entire MRI-enhanced lesion. Reda et al. ${ }^{16}$ used a same-day combined treatment in a patient harboring a cystic cerebellar hemangioblastoma; in that case GKS was followed by cyst aspiration. Those clinicians performed GKS first to avoid possible radiation injury to the medulla oblongata. They used a margin dose of $25 \mathrm{~Gy}$ at the $50 \%$ isodose line to cover the tumor, whereas other authors have used lower doses to treat hemangioblastomas. ${ }^{7,17}$

Niranjan et al..$^{13}$ performed GKS within 48 hours of cyst aspiration in 7 patients Four aspiration procedures were followed by GKS within 2 hours to capitalize on the reduced tumor volume. Stereotactic cyst aspiration and GKS are minimally invasive treatment modalities. The two procedures can be performed on the same day using one stereotactic frame application. In the present study almost all patients were transported to the MRI room immediately after cyst aspiration. Gamma Knife surgery normally began within 2 hours following aspiration, after the patients had undergone the second MRI examination and dose planning. No major complications were encountered during these procedures. Fourteen patients eventually required repeated aspiration and Ommaya reservoir placement. Thus for patients with cystic glial tumors or craniopharyngiomas, placement of an Ommaya reservoir during the first aspiration procedure should be considered.
Stereotactic cyst aspiration is rarely associated with the risk of hemorrhage, neurological deficits, seizures, or infection. Successful stereotactic cyst aspiration depends on appropriate patient selection and correct imaging interpretation. In particular, the determination of trajectory is the most important consideration before the procedure. ${ }^{8}$ Small hemorrhages are always self-limited; in most situations one only needs to allow the blood to drain out of the catheter. It is also important to calculate cyst volume carefully before aspiration and not to be too radical in suctioning.

\section{Conclusions}

The same-day combined stereotactic aspiration and GKS procedure was safe in patients with cystic brain tumors. Prompt symptom relief was achieved after cyst aspiration. The decrease in tumor volume made the following GKS more effective because a higher prescription dose could be administered with a lower possibility of radiation-induced side effects.

\section{Disclosure}

The authors report no conflict of interest concerning the materials or methods used in this study or the findings specified in this paper.

Author contributions to the study and manuscript preparation include the following. Conception and design: Xu. Acquisition of data: X Liu, Yu, Z Zhang, Y Zhang, Li, D Liu, Jia, Zheng. Analysis and interpretation of data: X Liu, Yu, Zheng. Drafting the article: X $\mathrm{Liu}, \mathrm{Yu}$. Reviewed submitted version of manuscript: Xu. Approved the final version of the manuscript on behalf of all authors: Xu. Administrative/technical/material support: Z Zhang, Y Zhang, Li, D Liu, Jia. Study supervision: $\mathrm{Xu}$.

\section{References}

1. Afra D, Norman D, Levin VA: Cysts in malignant gliomas. Identification by computerized tomography. J Neurosurg 53: 821-825, 1980

2. Baggenstos MA, Butman JA, Oldfield EH, Lonser RR: Role of edema in peritumoral cyst formation. Neurosurg Focus 22(5): E9, 2007

3. Franzin A, Vimercati A, Picozzi P, Serra C, Snider S, Gioia L, et al: Stereotactic drainage and Gamma Knife radiosurgery of cystic brain metastasis. J Neurosurg 109:259-267, 2008

4. Greenblatt SH, Rayport M, Savolaine ER, Harris JH, Hitchins MW: Computed tomography-guided intracranial biopsy and cyst aspiration. Neurosurgery 11:589-598, 1982

5. Gutin PH, Klemme WM, Lagger RL, MacKay AR, Pitts LH, Hosobuchi Y: Management of the unresectable cystic craniopharyngioma by aspiration through an Ommaya reservoir drainage system. J Neurosurg 52:36-40, 1980

6. Hood TW, McKeever PE: Stereotactic management of cystic gliomas of the brain stem. Neurosurgery 24:373-378, 1989

7. Kano H, Niranjan A, Mongia S, Kondziolka D, Flickinger JC, Lunsford LD: The role of stereotactic radiosurgery for intracranial hemangioblastomas. Neurosurgery 63:443-451, 2008

8. Kondziolka D, Firlik AD, Lunsford LD: Complications of stereotactic brain surgery. Neurol Clin 16:35-54, 1998

9. Kondziolka D, Lunsford LD, Flickinger JC: Stereotactic radiosurgery using the gamma knife: indications and results. Neurologist 3:45-52, 1997

10. Lohle PN, Wurzer HA, Seelen PJ, Kingma LM, Go KG: Cystic lesions accompanying extra-axial tumours. Neuroradiology 41:13-17, 1999 
11. Maiuri F: Cysts with mural tumor nodules in the cerebral hemispheres. Neurosurgery 22:703-706, 1988

12. Maldaun MV, Suki D, Lang FF, Prabhu S, Shi W, Fuller GN, et al: Cystic glioblastoma multiforme: survival outcomes in 22 cases. J Neurosurg 100:61-67, 2004

13. Niranjan A, Witham T, Kondziolka D, Lunsford LD: The role of stereotactic cyst aspiration for glial and metastatic brain tumors. Can J Neurol Sci 27:229-235, 2000

14. Palma L, Guidetti B: Cystic pilocytic astrocytomas of the cerebral hemispheres. Surgical experience with 51 cases and long-term results. J Neurosurg 62:811-815, 1985

15. Park CK, Kim DC, Park SH, Kim JE, Paek SH, Kim DG, et al: Microhemorrhage, a possible mechanism for cyst formation in vestibular schwannomas. J Neurosurg 105:576-580, 2006

16. Reda WA, Hay AA, Ganz JC: A planned combined stereotactic approach for cystic intracranial tumors. Report of two cases. J Neurosurg 97 (5 Suppl):610-612, 2002
17. Sayer FT, Nguyen J, Starke RM, Yen CP, Sheehan JP: Gamma knife radiosurgery for intracranial hemangioblastomas-outcome at 3 years. World Neurosurg 75:99-105, 2011

18. Yamanaka Y, Shuto T, Kato Y, Okada T, Inomori S, Fujino H, et al: Ommaya reservoir placement followed by Gamma Knife surgery for large cystic metastatic brain tumors. J Neurosurg 105 Suppl:79-81, 2006

Manuscript submitted May 15, 2012.

Accepted July 2, 2012.

Please include this information when citing this paper: DOI: 10.3171/2012.7.GKS121019.

Address correspondence to: Desheng Xu, M.D., Gamma Knife Center, Department of Neurosurgery, Second Hospital of Tianjin Medical University, 23 Pingjiang Road, Hexi District, Tianjin 300211, People’s Republic of China. email: tjxudesheng@163.com. 